\title{
Development and evaluation of the refined zenith tropospheric delay (ZTD) models
}

\author{
Fei Yang ${ }^{1 *} \mathbb{D}$, Xiaolin Meng ${ }^{2}$, Jiming Guo ${ }^{3}$, Debao Yuan ${ }^{1}$ and Ming Chen ${ }^{4}$
}

\begin{abstract}
The tropospheric delay is a significant error source in Global Navigation Satellite System (GNSS) positioning and navigation. It is usually projected into zenith direction by using a mapping function. It is particularly important to establish a model that can provide stable and accurate Zenith Tropospheric Delay (ZTD). Because of the regional accuracy difference and poor stability of the traditional ZTD models, this paper proposed two methods to refine the Hopfield and Saastamoinen ZTD models. One is by adding annual and semi-annual periodic terms and the other is based on Back-Propagation Artificial Neutral Network (BP-ANN). Using 5-year data from 2011 to 2015 collected at 67 GNSS reference stations in China and its surrounding regions, the four refined models were constructed. The tropospheric products at these GNSS stations were derived from the site-wise Vienna Mapping Function 1 (VMP1). The spatial analysis, temporal analysis, and residual distribution analysis for all the six models were conducted using the data from 2016 to 2017. The results show that the refined models can effectively improve the accuracy compared with the traditional models. For the Hopfield model, the improvement for the Root Mean Square Error (RMSE) and bias reached 24.5/49.7 and 34.0/52.8 mm, respectively. These values became 8.8/26.7 and 14.7/28.8 $\mathrm{mm}$ when the Saastamoinen model was refined using the two methods. This exploration is conducive to GNSS navigation and positioning and GNSS meteorology by providing more accurate tropospheric prior information.
\end{abstract}

Keywords: GNSS, Tropospheric delay, ZTD, Refined model, ANN

\section{Introduction}

During propagating through the neutral atmosphere, Global Navigation Satellite System (GNSS) signals from a satellite to a receiver will be delayed and bent due to their interaction with dry gases and water particles, which is called tropospheric delay (Bevis et al., 1992; Yao et al., 2018). It is a significant error source in GNSS positioning and navigation, for the delay varies from 2 to $20 \mathrm{~m}$ depending on the elevation angle of a satellite (Chen et al., 2020; Penna et al., 2001). We generally project the tropospheric delay into zenith direction by using a mapping function and utilize the Zenith Tropospheric Delay (ZTD) to describe the tropospheric influence on

\footnotetext{
*Correspondence: yangfei@cumtb.edu.cn

${ }^{1}$ College of Geoscience and Surveying Engineering, China University

of Mining and Technology-Beijing, 100083 Beijing, China

Full list of author information is available at the end of the article
}

the signal propagation. An accurate ZTD is not only an important parameter for GNSS navigation and positioning (Duan et al., 1996; Meng, 2002; Zhang et al., 2017; Zumberge et al., 1997), but also the basis for retrieving Precipitable Water Vapor (PWV) in GNSS meteorology (Li et al., 2014; Yang et al., 2020a; Zheng et al., 2018). A stable and accurate ZTD model is necessary to meet these requirements.

Two types of ZTD models are commonly used: (1) ZTD models with the measured meteorological parameters at a site, such as Hopfield model, Saastamoinen model and Black model, which can achieve centimeter-level accuracy by inputting accurately measured meteorological parameters (Hopfield, 1969; Black \& Eisner, 1984; Saastamoinen, 1972); (2) the empirical ZTD models, which feedback only by the location of a site and time of interest. Some empirical models, such as GZTD series (Yang et al., 2020b; Yao et al., 2013, 2016) 
and IGGtrop series (Li et al., 2012, 2015, 2018), are established by using the trend analysis on long-term ZTD values. The other empirical models, such as GPT series (Boehm et al., 2007; Bohm et al., 2015; Lagler et al., 2013; Landskron \& Boehm, 2018), are first building the models of various meteorological quantities, and then estimating the ZTD with these estimated meteorological parameters and the formula of Saastamoinen model, Hopfield model, and other models. Thus, the ZTDs estimated by the above two types of empirical models have generally poorer results than those with Saastamoinen and Hopfield model based on the measured meteorological data. However, several studies confirmed that the Saastamoinen and Hopfield models tend to be poor when using the regional meteorological data in a local area (Yang et al., 2020c, 2021). The obvious regional differences in the accuracy with the Saastamoinen and Hopfield models are due to the fact that they were constructed based on global mean meteorological data and global climate analysis, which makes it difficult to describe the ZTD characteristics in certain areas. Therefore, it is necessary to perform the regional refinement of the ZTD models, which can not only optimize the performance of the corresponding parameter models in a specific area, but also improve the accuracy of empirical ZTD models.

In this paper, two regional refined methods are proposed for the Hopfield and Saastamoinen models. The first method introduces the annual and semi-annual periodic terms in the Saastamoinen and Hopfield models and utilizes the least-squares fitting method to establish the regional refined models. The second method adopts Back-Propagation Artificial Neural Network (BP-ANN) to perform error compensation for the Saastamoinen and Hopfield models.

\section{The refined models}

Hopfield and Saastamoinen models are the most used ZTD models, which use the surface pressure, temperature, and water vapor pressure to estimate ZTD above a specific site. The Hopfield ZTD model is expressed as follows (Hopfield, 1969):

$$
\begin{aligned}
\mathrm{ZTD}_{H}= & 1.552 \times 10^{-5} \times P_{s} / T_{s} \cdot\left(h_{d}-h_{s}\right) \\
& +0.07465 \times e_{s} / T_{s}^{2} \cdot\left(h_{w}-h_{s}\right)
\end{aligned}
$$

where $\mathrm{ZTD}_{H}$ denotes the ZTD estimates of Hopfield model, $P_{s}, T_{s}$ and $e_{s}$ represent pressure (in $\mathrm{hPa}$ ), temperature (in $\mathrm{K}$ ), and water vapor pressure (in $\mathrm{hPa}$ ), respectively, $h_{s}$ denotes the height of a site above the mean sea level,. $h_{d}=40,136+148.72\left(T_{s}-273.15\right) \mathrm{m}$ and $h_{w}=11,000 \mathrm{~m}$ are the height of tropopause and wet tropopause, respectively.
The Saastamoinen ZTD model is represented by the following equation (Saastamoinen, 1972):

$$
\operatorname{ZTD}_{S}=0.002277 / f\left(\varphi, h_{s}\right) \times\left[P_{s}+\left(1255 / T_{s}+0.05\right) \times e_{s}\right](2)
$$

where $\mathrm{ZTD}_{S}$ denotes the ZTD estimates of the Saastamoinen model, $\varphi$ represents the latitude of the site, and $f$ is the correction of gravitational acceleration caused by the rotation of the Earth, which can be calculated by the following formula:

$$
f\left(\varphi, h_{s}\right)=1-0.00266 \times \cos 2 \varphi-0.00028 \times h_{s}
$$

The research on the temporal and spatial distributions of ZTD found that ZTD has obvious annual and semiannual variations (Mao et al. 2013; Myers et al. 2013). The idea of compensating the Hopfield model by adding annual and semi-annual periodic terms was utilized (Yang et al. 2020c). In this paper, we refined the Hopfield and Saastamoinen models, called the Hop-r1 and Saas-r1 models, which are expressed as follows:

$$
\begin{aligned}
\mathrm{ZTD}_{\mathrm{Hr} 1}= & \mathrm{ZTD}_{H}+a_{11} \cos \left(2 \pi \frac{\text { doy }}{365.25}\right) \\
& +a_{12} \sin \left(2 \pi \frac{\text { doy }}{365.25}\right) \\
& +a_{13} \cos \left(4 \pi \frac{\text { doy }}{365.25}\right) \\
& +a_{14} \cos \left(4 \pi \frac{\text { doy }}{365.25}\right)+c_{1} \\
\mathrm{ZTD}_{\mathrm{Sr} 1}= & \mathrm{ZTD}_{S}+a_{21} \cos \left(2 \pi \frac{\mathrm{doy}}{365.25}\right) \\
& +a_{22} \sin \left(2 \pi \frac{\text { doy }}{365.25}\right) \\
& +a_{23} \cos \left(4 \pi \frac{\mathrm{doy}}{365.25}\right) \\
& +a_{24} \cos \left(4 \pi \frac{\mathrm{doy}}{365.25}\right)+c_{2}
\end{aligned}
$$

where $\mathrm{ZTD}_{\mathrm{hr} 1}$ and $\mathrm{ZTD}_{\mathrm{sr} 1}$ denote the ZTD estimates of the refined Hopfield and Saastamoinen models, doy represents the day of year, $\left(a_{11}, a_{12}\right)$ and $\left(a_{21}, a_{22}\right)$ are the annual amplitudes for the two refined models, $\left(a_{13}, a_{14}\right)$ and $\left(a_{23}, a_{24}\right)$ are their semi-annual amplitudes, and $c_{1}$ and $c_{2}$ denote their constant terms. To calculate the ten coefficients in the above two refined models, we utilized the accurate tropospheric products of the GNSS stations provided by the site-wise Vienna Mapping Function 1 (VMF1) and adopted the least-squares method.

An Artificial Neural Network (ANN) is designed to simulate the way with which the human brain analyzes and process information, which is widely used in 
classification, regression, and in the geoscience field (Yang et al. 2020d). Composed of an input layer, an output layer and one or more hidden layers, the ANN can efficiently handle the relations between input and output variables and produce better results as enough data become available. In this paper, we utilized the BP-ANN to construct the relationship between the ZTD estimated with the models and the true ZTD values to achieve the purpose of ZTD error compensation. Specially, four input parameters are selected in this research, including temperature, pressure, water vapor pressure, and ZTD estimates of parameter models. The output parameter is the true ZTD value. We refined Hopfield and Saastamoinen models based on the BP-ANN, which are called Hop-r2 and Saas-r2 models, respectively. Figure 1 is a flowchart showing the basic process of constructing these two models based on BP-ANN.

The site-wise VMF1 tropospheric products contain the meteorological parameters and the true ZTD values of the selected GNSS stations, therefore, it can provide the dataset for BP-ANN training. We divide the datasets into a training set and a validation set, accounting for 75 and $25 \%$ of the total data sets, respectively. The function of training set and validation set are to adjust the weights on the neural network and to minimize overfitting, respectively. The BP-ANN structure used for the two refined models are as follows: four nodes in the input layers, which is the same as the number of input parameters. A single node in the output layer is the true ZTD value. There are two hidden layers with four nodes. The used training and activation functions are LevenbergMarquardt and hyperbolic tangent, respectively. The values of $6000,0.01$ and 0.001 were selected for the maximum training number, learning rate and error threshold, respectively.

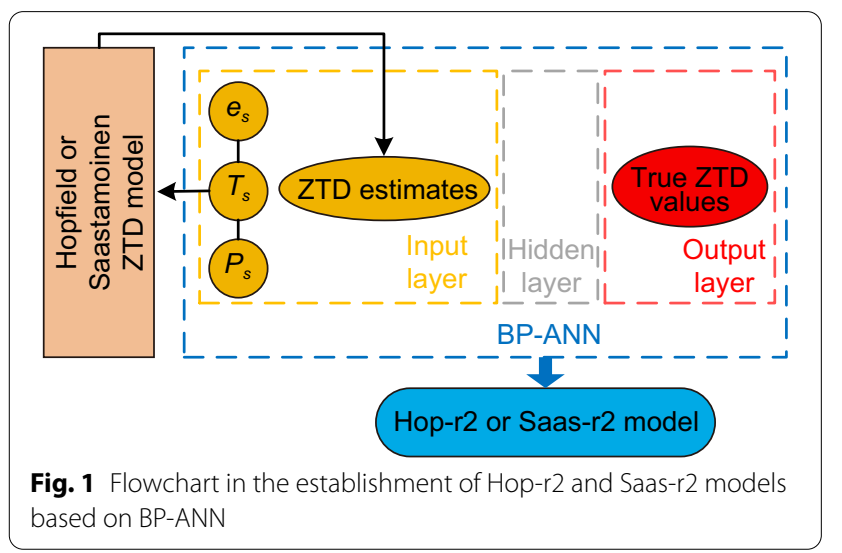

\section{Analysis of the refined ZTD models}

The 5-year data from 2011 to 2015 collected at 67 GNSS stations in China and surrounding regions are used to construct the above mentioned four refined models. To assess the performance of the proposed ZTD models, we evaluated the ZTD values estimated with different models using the true ZTD values of year 2016-2017 provided by site-wise VMF1 as references. Thus, there are six models for estimating ZTD in the comparisons, including the Hopfield model, the refined Hopfield model with periodic terms (Hop-r1), the refined Hopfield model based on BP-ANN (Hop-r2), the Saastamoinen model, the refined Saastamoinen model with periodic terms (Saas-r1), and the refined Saastamoinen model based on BP-ANN (Saas-r2). Two statistical quantities, i.e., bias and Root Mean Square Error (RMSE), are chosen as the criteria to assess the performance of each model.

The ZTD estimates of all stations in the research area are calculated by these six models and compared with the references at the corresponding time. Figure 2 represents the maps of RMSE, which shows the different performances of the six models at each site. It shows the influence of site latitude on RMSE, that is, the RMSE is always small at high latitudes and becomes large at the middle and low latitudes. The two traditional models perform poorly, especially the Hopfield model. All four refined models can improve the accuracy compared with the traditional models, as indicated by the color change of the points in the figure. It is obvious that both refined Hopfield models still have some stations with poor accuracy. In this comparison, the refined Saastamoinen models do not show this phenomenon, indicating that they are better than the refined Hopfield models. It is observed that the refined models based on the BP-ANN perform better than the those with periodic terms, indicating that the advantages of the second refinement method.

The maps of bias for the six models are illustrated in Fig. 3. The negative bias appears at each site for the two traditional models. The absolute value of bias increases as the latitude decreases for the Saastamoinen model, while the bias is always a large negative value at most of the stations for the Hopfield model. After the refinement, the biases for the four refined models at each site are closer to 0 with small positive value at some stations. Note that the Hop-r1 model hardly shows an improvement at some stations. One can observe that the refined models based on the BP-ANN give the best results, which is similar to that of Fig. 2.

The mean RMSE and bias, as well as their maximum and minimum values, of the differences between the ZTD estimated with the six models and the referenced ZTD at all stations are summarized in Table 1. For RMSE, the Hopfield model performs worst with the values of 


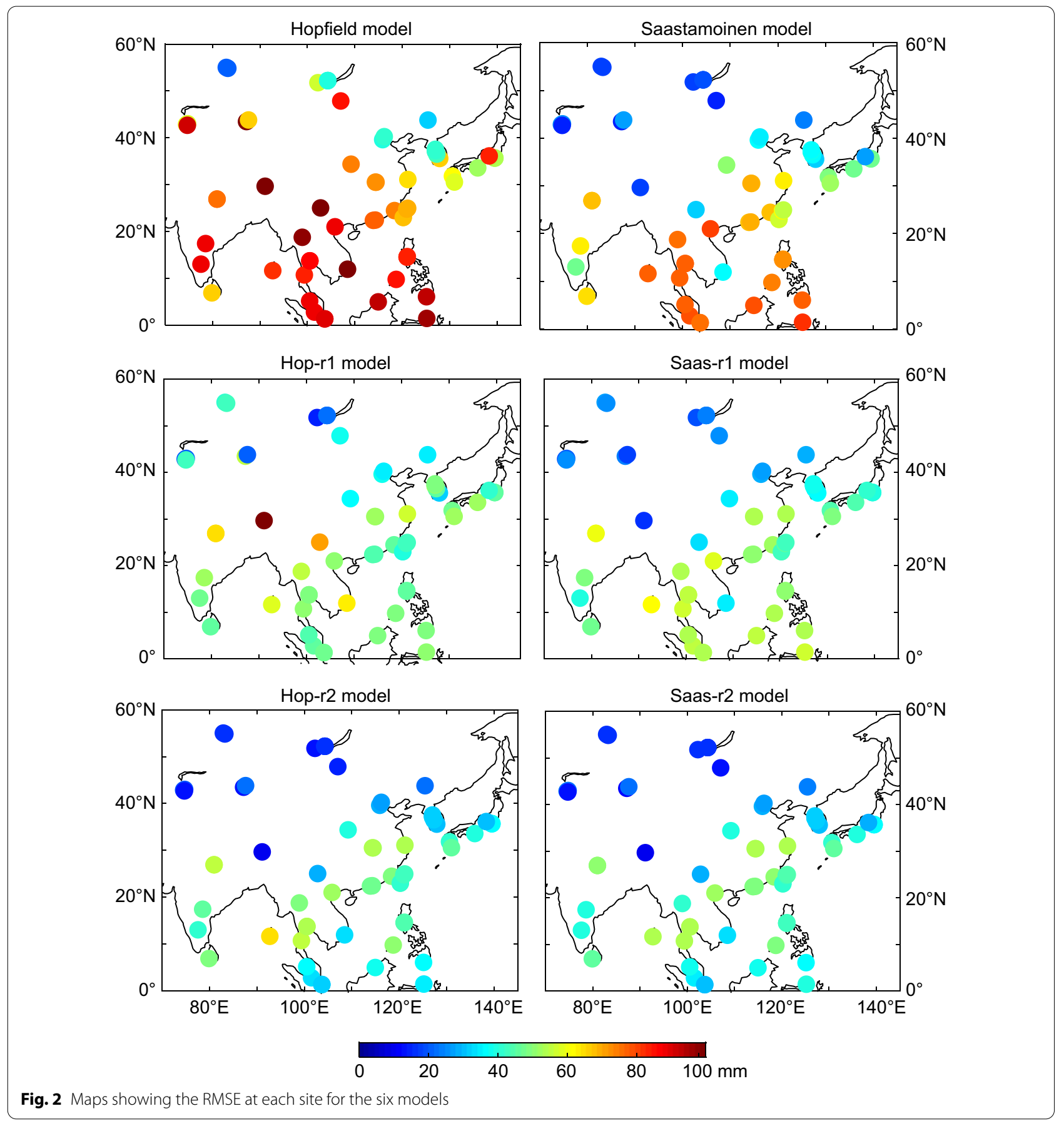

70.5/161.0/20.5 $\mathrm{mm}$ for the mean, maximum, and minimum, respectively. The two refined models significantly improve the results, with the mean RMSE reduced from 70.5 to $46.0 \mathrm{~mm}$ and $36.5 \mathrm{~mm}$, an improvement by about 35 and $48 \%$, respectively. The Saas-r2 model achieves the best performance, i.e., the mean, maximum, and minimum of RMSE are 36.0, 54.1 and $9.1 \mathrm{~mm}$, respectively.
For bias, we can obtain the same conclusions from theses statistical values.

Considering the complex topography of the research area, the changes in the mean RMSE and the bias with the height of a site are analyzed for the six models, which are shown in Figs. 4 and 5. It is obvious that the heigh has a great influence on the accuracy of the Hopfield model, 

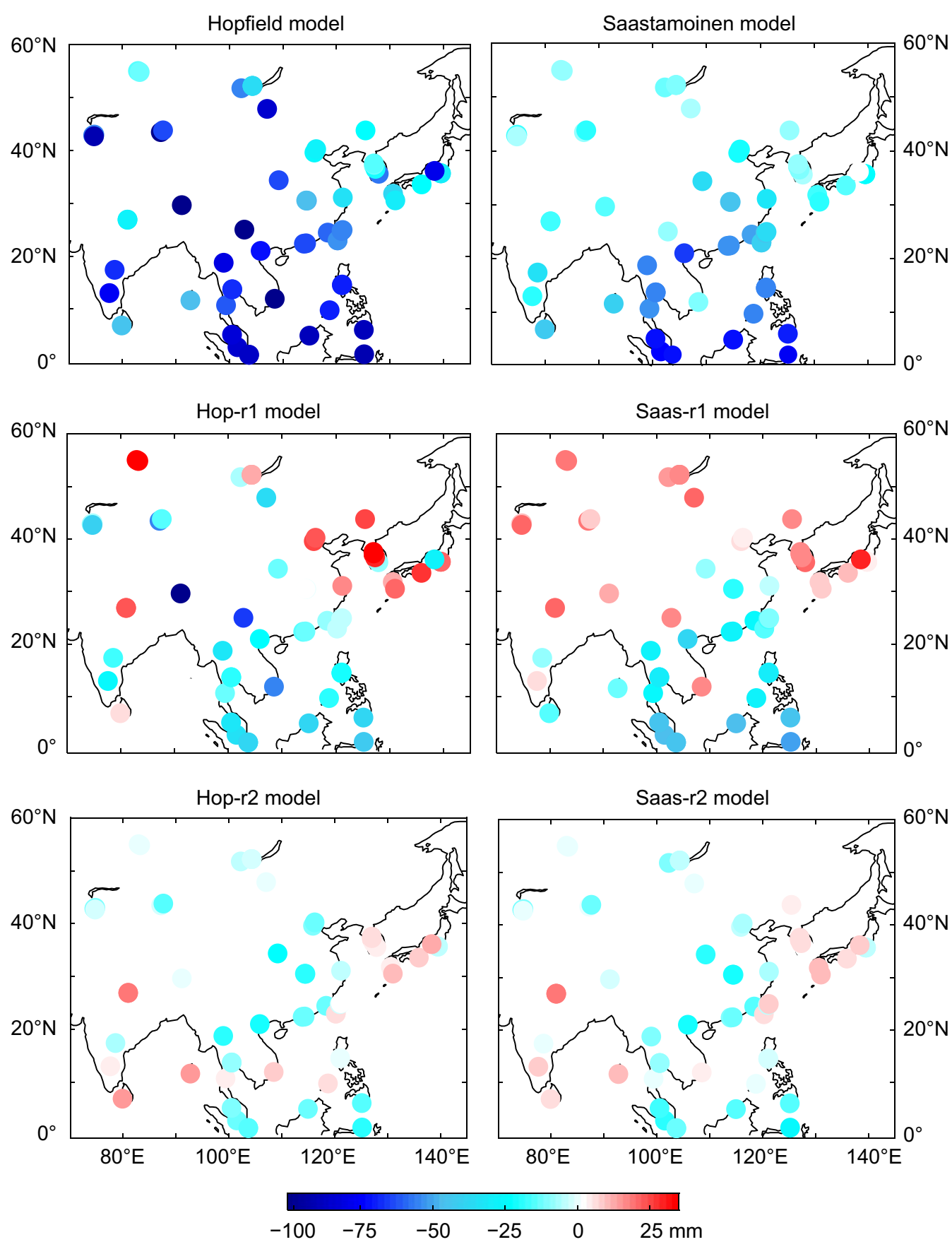

Fig. 3 Maps showing the bias at each site for six models

that is, the larger the height of the site is, the larger the RMSE and the negative bias will be. After the refinement, the Hop-r1 model improves this phenomenon, but its accuracy is still affected by the site height. The Hopr2 model performs well, and its accuracy is not affected by the height of a site. For the Saastamoinen model, its RMSE and bias are not affected by the height of a site.
Moreover, the two refined Saastamoinen models improve the performance at each site, especially the Saas-r2 model

To analyze the variation of the ZTD values estimated with the six models in different seasons and months, the bias and RMSE for all stations in the research area are calculated monthly and seasonally, as shown in Figs. 6 and 7. It can be seen that the ZTD biases of the Hopfield 
Table 1 Mean RMSE and bias of the ZTD differences for the six models at all stations

\begin{tabular}{|c|c|c|c|c|c|c|}
\hline \multirow[t]{2}{*}{ Names of the model } & \multicolumn{3}{|c|}{ Results of RMSE (mm) } & \multicolumn{3}{|c|}{ Results of bias (mm) } \\
\hline & Mean & Maximum & Minimum & Mean & Maximum & Minimum \\
\hline Hopfield & 70.5 & 161.0 & 20.5 & -55.0 & -11.9 & -159.6 \\
\hline Hop_r1 & 46.0 & 110.7 & 14.6 & -5.3 & 37.8 & -110.0 \\
\hline Hop_r2 & 36.5 & 64.1 & 9.4 & -2.2 & 20.1 & -23.0 \\
\hline Saastamoinen & 50.7 & 81.8 & 14.1 & -31.4 & 2.6 & -76.3 \\
\hline Saas_r1 & 41.9 & 61.1 & 13.6 & -4.7 & 29.3 & -49.7 \\
\hline Saas_r2 & 36.0 & 54.1 & 9.1 & -2.7 & 20.0 & -25.2 \\
\hline
\end{tabular}

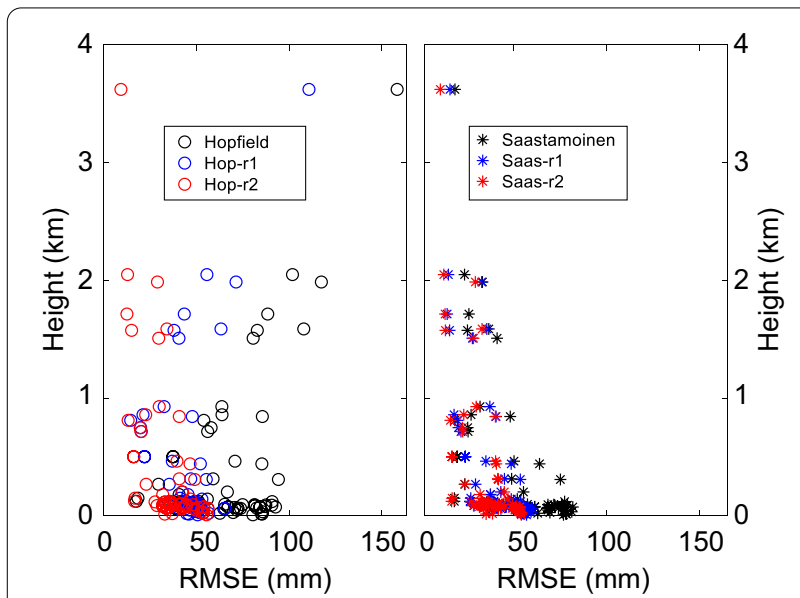

Fig. 4 Change in the RMSE with the height of a site for the six models

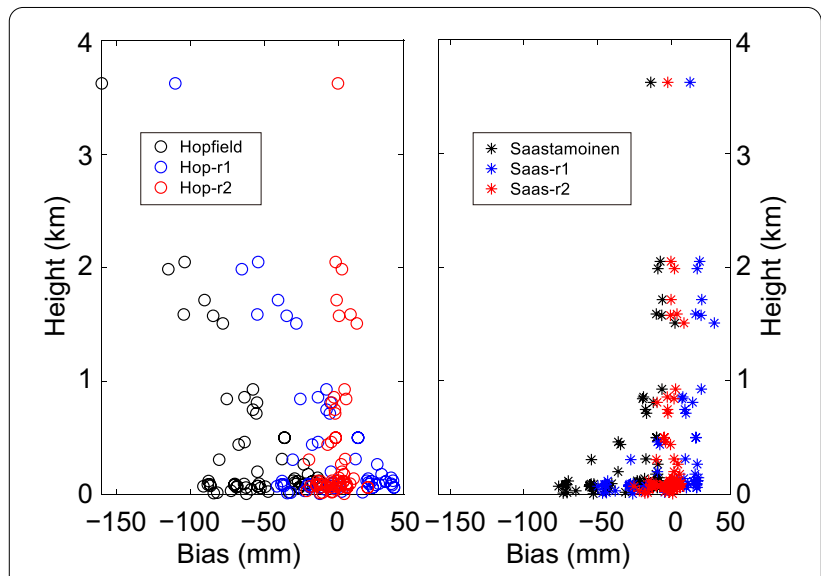

Fig. 5 Change in the bias with the height of a site for the six models

and Saastamoinen models show apparent seasonal effects with the peaks in summer, and the maximum negative biases are in July which are -83.5 and $-54.2 \mathrm{~mm}$, respectively. It indicates that the water vapor in the

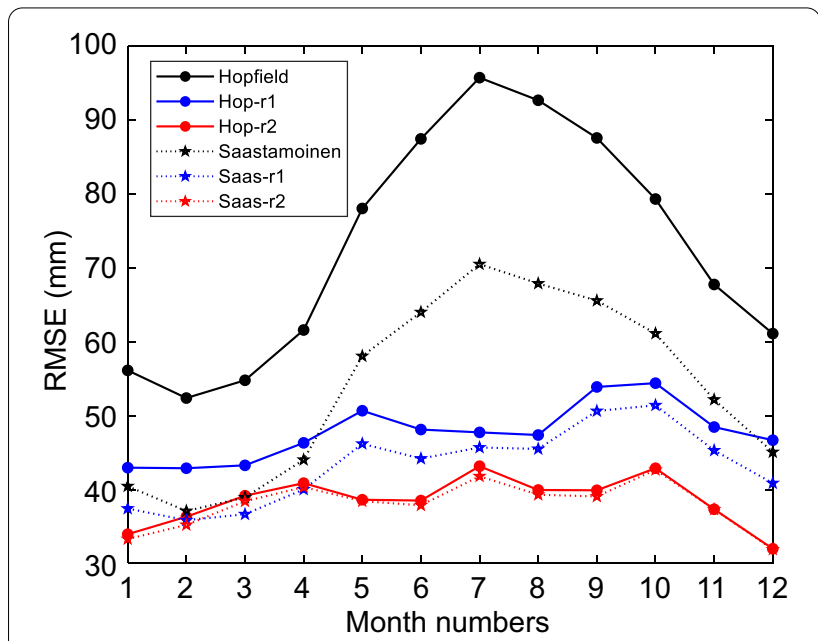

Fig. 6 Monthly mean RMSE of the stations for six models

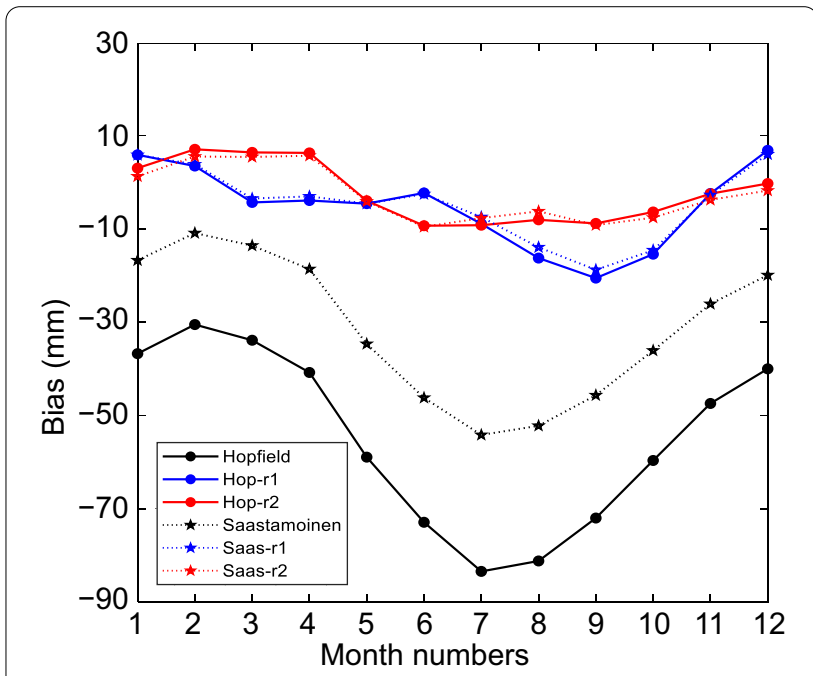

Fig. 7 Monthly mean bias of the stations for six models

research area changes greatly in summer, the ZTD estimates with the two traditional models are smaller than the referenced ZTD, and therefore implementation of 
error compensation is necessary. Correspondingly, their RMSE experiences an increase from Spring to Summer, and then a decrease from Summer to Autumn with the maximum values of $95.8 \mathrm{~mm}$ and $70.6 \mathrm{~mm}$, respectively. For the four refined models, their monthly mean biases and RMSEs have no obvious seasonal changes, and the monthly fluctuations are also small. For example, the maximum and minimum monthly mean RMSEs of the Hop-r2 model appear in July and December, and their values are $43.2 \mathrm{~mm}$ and $32.1 \mathrm{~mm}$, respectively. For the Saas-r2 model, these values are $42.7 \mathrm{~mm}$ and $31.9 \mathrm{~mm}$ in October and December, respectively.

Further, the performance of the six models to estimate ZTD at different Coordinated Universal Time (UTC) epochs are analyzed. Their RMSE distributions at the four UTC epochs are illustrated in Fig. 8. Based on the accuracy from low to high the six models at each UTC epoch are ranked as the Hopfield model, Saastamoinen model, Hop-r1 model, Saas-r1 model, Hop-r2 model, and Saas-r2 model. The accuracy for the Hopfield and Saastamoinen models is similar at different UTC time. The RMSE of the four refined models at UTC 6:00 and UTC 12:00 are slightly smaller than those at UTC 0:00 and UTC 18:00. The Saas-r2 model has the best performance at each UTC epoch, and the values at these UTC epochs are $37.8,34.4,34.7$ and $36.9 \mathrm{~mm}$, respectively.

The histogram of the ZTD residuals, namely the differences between the model-derived ZTDs and the referenced ZTDs, is shown in Fig. 9. One can see that most of the ZTD residuals calculated with the Hopfield and Saastamoinen models are less than 0 . The percentages of the ZTD residuals larger than $-100 /-50 \mathrm{~mm}$ are

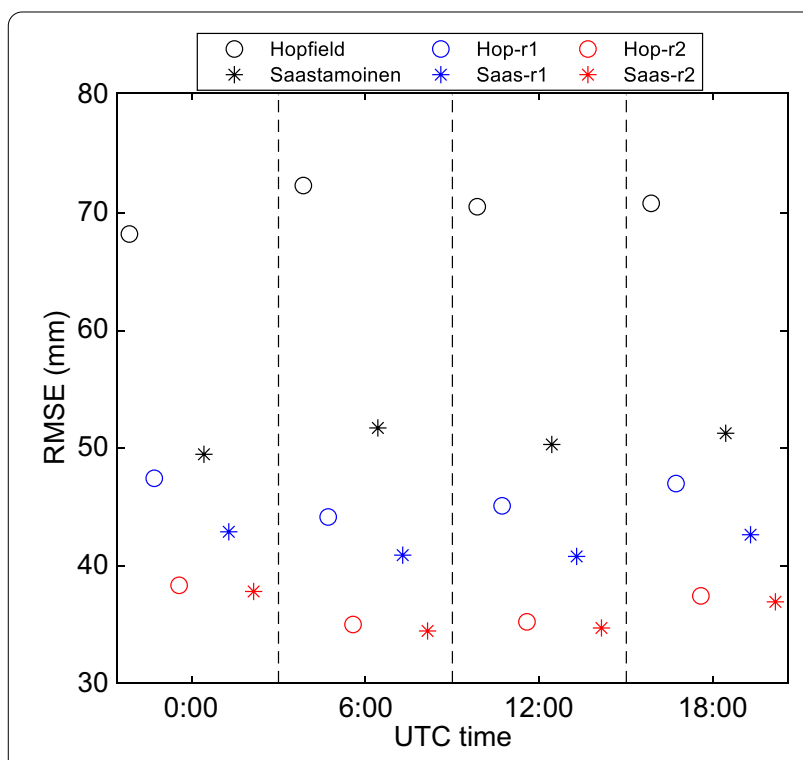

Fig. 8 The performance of the six models at different UTC epochs
$20.5 \% / 52.3$ and $8.7 \% / 32.5 \%$ for the Hopfield and Saastamoinen models, respectively, indicating that the residual distribution for the Saastamoinen model is slightly better than the Hopfield model. After the refinement, the Hopr1 makes the ZTD residual distribution closer to the normal distribution, and the Saas-r2 model leads more ZTD residuals closer to zero. The two refined models based on the BP-ANN achieve the best ZTD residuals distributions, which basically follow the normal distribution and make most of the ZTD residuals concentrated around 0 $\mathrm{mm}$. For example, the percentages of the ZTD residuals in the range of -10 to $10 \mathrm{~mm}$ are 29.0 and $28.6 \%$ for the Hop-r2 model and the Saas-r2 model, respectively. When the range changes to -50 to $50 \mathrm{~mm}$, these percentages become 81.5 and $82.4 \%$ for the two refined models.

Moreover, the Standard Deviation (SD) of the ZTD residuals is computed for the six models. These values are $50.4 \mathrm{~mm}$ and $45.3 \mathrm{~mm}$ for the Hopfield and Saastamoinen models, respectively. The two corresponding refined models improves the SD of the Hopfield model by 6.7 and $23.2 \%$, respectively. The Saas-r1 and Saas-r2 models achieve SD values of $43.1 \mathrm{~mm}$ and $38.0 \mathrm{~mm}$, an improvement by about 4.9 and $16.1 \%$ over the Saastamoinen model.

\section{Conclusions}

To refine the Saastamoinen model and the Hopfield model, two methods were introduced, namely the method by adding annual and semi-annual periodic terms and the method based on the BP-ANN. Therefore, four refined ZTD models are established using the ZTD products provided by the site-wise VMF1. The comprehensive comparisons between the four refined models and the two traditional models are conducted using the 2 years data derived from the site-wise VMF1 tropospheric products. From the spatial analysis the accuracy for the two traditional models shows a spatial difference and is affected by the latitude of a site. Moreover, the accuracy of the Hopfield model becomes worse as an increase in the site height. The refined models can effectively overcome the above problems, especially the refined models based on the BP-ANN. For example, the mean bias and RMSE of the Hop-r2 and Saas-r2 model are - 2.2/36.5 $\mathrm{mm}$ and $-2.7 / 36.0 \mathrm{~mm}$, respectively. From the temporal analysis of the model accuracy, the two traditional models appear obvious seasonal effect and have the worst performance in summer. The four refined models can eliminate the seasonal influence of the estimated ZTD, and the monthly fluctuation also becomes very small. The accuracies of all six models are not affected by different UTC epochs, and the Saas-r2 model has the best performance at each UTC epoch. From the analysis of 

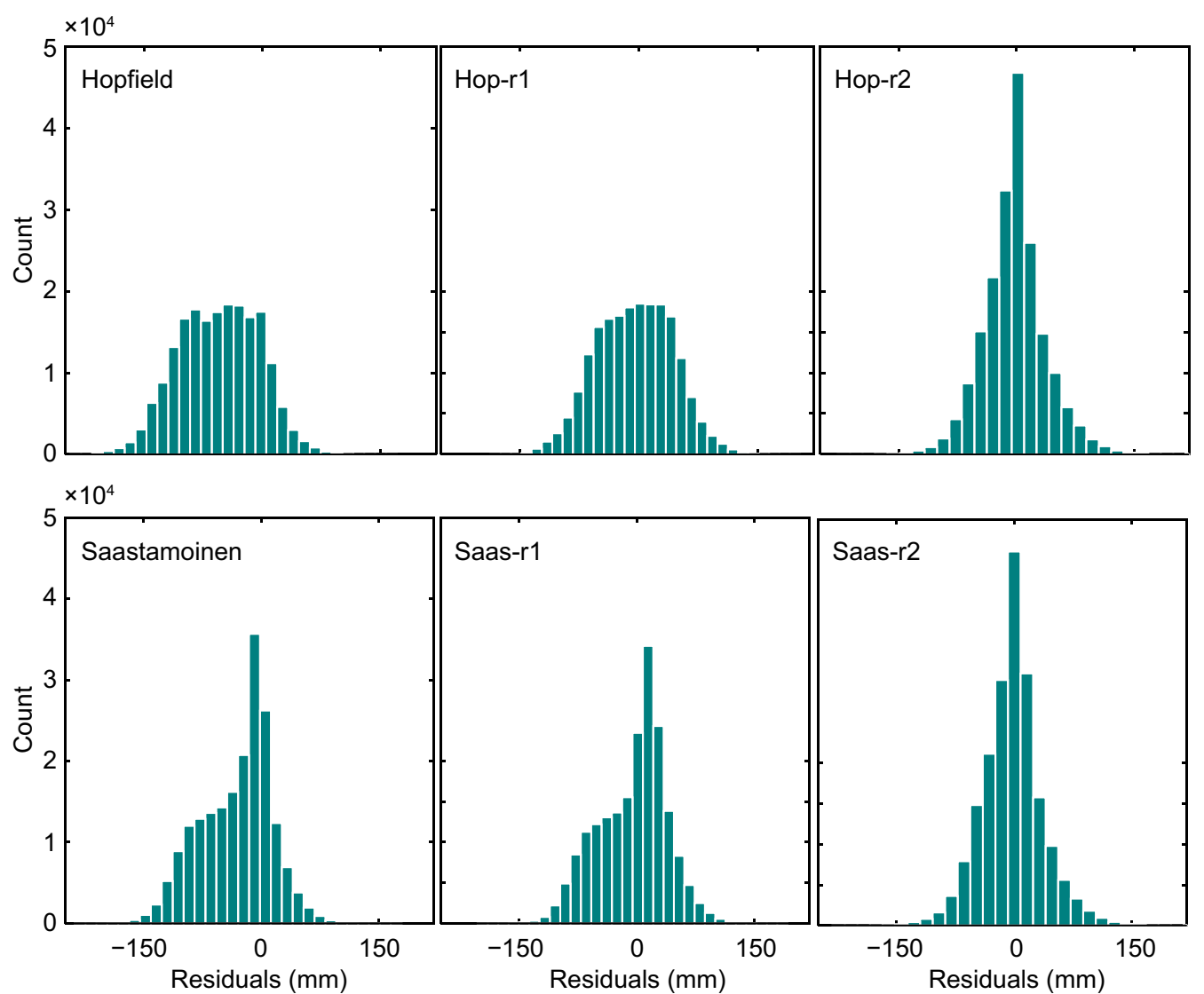

Fig. 9 Histogram of the residuals between the ZTD derived from six models and the referenced ZTD

residual distributions, the refined models can improve the residual distributions compared with the traditional models, especially the models based on the BP-ANN, which make the ZTD residuals follow the normal distribution and concentrated around zero. We constructed the refined models in China and surrounding regions, which can improve the accuracy of estimated ZTD in this region, and therefore provide more accurate information on troposphere for the research on GNSS navigation and positioning and GNSS meteorology. In further research, the refined models that are better suitable for local areas should be explored, such as constructing the coefficients of the refined models in the form of dense grids.

\section{Acknowledgements}

The authors would like to thank the research group of Advanced Geodesy of TU Vienna for providing the VMF1 products. We thank all anonymous reviewers for their valuable, constructive, and prompt comments.

\section{Authors' contributions}

Conceptualization, FY and XM; methodology, FY; data curation, JG, DY, and MC; validation, FY, DY and MC; formal analysis, FY; resources, JG, and MC.; writing — original draft preparation, FY; writing — review and editing, FY, XM, JG, DY and MC. All authors read and approved the final manuscript.

\section{Funding}

This research was supported by the Fundamental Research Funds for the Central Universities (No. 2021XJDC01), the State Key Laboratory of Resources and Environmental Information System, the Key Laboratory of Geospace Environment and Geodesy, Ministry of Education, Wuhan University (No. 19-02-08), the Guangxi Key Laboratory of Spatial Information and Geomatics (No. 19-050-11-01), the National Natural Science Foundation of China (Nos. 41804038, 42001368).

\section{Availability of data and materials}

The raw data were provided by the VMF Data Server. The other data used in this study are available from the corresponding author upon request.

\section{Declarations}

\section{Competing interests}

The authors declare that they have no competing interests.

\section{Author details}

${ }^{1}$ College of Geoscience and Surveying Engineering, China University of Mining and Technology-Beijing, 100083 Beijing, China. ${ }^{2}$ Global Geospatial Engineering Ltd., The Sino-UK Geospatial Engineering Centre, 18 Radford Bridge Road, Nottingham NG8 1NA, UK. ${ }^{3}$ School of Geodesy and Geomatics, Wuhan University, 129 Luoyu Road, Wuhan 430079, China. ${ }^{4}$ National Geomatics Center of China, Beijing 100830, China. 
Received: 19 April 2021 Accepted: 25 August 2021

Published online: 04 October 2021

\section{References}

Bevis, M., Businger, S., Herring, T. A., Rocken, C., Anthes, R. A., \& Ware, R. H. (1992). GPS meteorology: Remote sensing of atmospheric water vapor using the global positioning system. Journal of Geophysical Research: Atmospheres, 97, 15787-15801. https://doi.org/10.1029/92jd01517.

Black, H. D., \& Eisner, A. (1984). Correcting satellite Doppler data for tropospheric effects. Journal of Geophysical Research: Atmospheres, 89(D2), 2616-2626. https://doi.org/10.1029/JD089iD02p02616.

Boehm, J., Heinkelmann, R., \& Schuh, H. (2007). Short Note: A global model of pressure and temperature for geodetic applications. Journal of Geodesy, 81, 679-683. https://doi.org/10.1007/s00190-007-0135-3.

Böhm, J., Möller, G., Schindelegger, M., Pain, G., \& Weber, R. (2015). Development of an improved empirical model for slant delays in the troposphere (GPT2w). GPS Solutions, 19, 433-441. https://doi.org/10.1007/ s10291-014-0403-7.

Chen, P., Ma, Y., Liu, H., \& Zheng, N. (2020). A new global tropospheric delay model considering the spatiotemporal variation characteristics of ZTD with altitude coefficient. Earth and Space Science. https://doi.org/10.1029/ 2019 ea000888.

Duan, J., Bevis, M., Fang, P., et al. (1996). GPS meteorology: Direct estimation of the absolute value of precipitable water. Journal of Applied Meteorology and Climatology, 35, 830-838. https://doi.org/10.1175/1520-0450(1996) 0352.0.CO;2.

Hopfield, H. S. (1969). Two-quartic tropospheric refractivity profile for correcting satellite data. Journal of Geophysical Research, 74(18), 4487-4499. https://doi.org/10.1029/JC074i018p04487.

Lagler, K., Schindelegger, M., Böhm, J., Krásná, H., \& Nilsson, T. (2013). GPT2: Empirical slant delay model for radio space geodetic techniques. Geophysical Research Letter, 40, 1069-1073. https://doi.org/10.1002/grl.50288.

Landskron, D., \& Boehm, J. (2018). VMF3/GPT3: Refined discrete and empirical troposphere mapping functions. Journal of Geodesy, 92, 349-360. https:// doi.org/10.1007/s00190-017-1066-2.

Li, W., Yuan, Y., Ou, J., Chai, Y., Li, Z., Liou, Y., \& Wang, N. (2015). New versions of the BDS/GNSS zenith tropospheric delay model IGGtrop. Journal of Geodesy, 89, 73-80. https://doi.org/10.1007/s00190-014-0761-5.

Li, W., Yuan, Y., Ou, J., \& He, Y. (2018). IGGtrop_SH and IGGtrop_rH: Two improved empirical tropospheric delay models based on vertical reduction functions. IEEE Transactions on Geoscience and Remote Sensing, 56, 5276-5288. https://doi.org/10.1109/tgrs.2018.2812850.

Li, W., Yuan, Y., Ou, J., Li, H., \& Li, Z. (2012). A new global zenith tropospheric delay model IGGtrop for GNSS applications. Chinese Science Bulletin, 57, 2132-2139. https://doi.org/10.1007/s11434-012-5010-9.

Li, X., Dick, G., Ge, M., Heise, S., Wickert, J., \& Bender, M. (2014). Real-time GPS sensing of atmospheric water vapor: Precise point positioning with orbit, clock, and phase delay corrections. Geophysical Research Letter, 41(10), 3615-3621. https://doi.org/10.1002/2013GL058721.

Mao, J., Zhu, C., \& Guo, J. (2013). A new global tropospheric zenith delay model. Geomatics and Information Science of Wuhan University, 38(6), $684-688$

Meng, X. (2002). Real-time deformation monitoring of bridges using GPS/accelerometers. The University of Nottingham.

Myers, T., Maibach, E., \& Roser, C. (2013). The relationship between personal experience and belief in the reality of global warming. Nature Climate Change, 3(4), 343-347. https://doi.org/10.1038/NCLIMATE1754.
Penna, N., Dodson, A., \& Chen, W. (2001). Assessment of EGNOS tropospheric correction model. Journal of Navigation, 54, 37-55. https://doi.org/10. 1017/s0373463300001107.

Saastamoinen, J. (1972). Atmospheric correction for the troposphere and stratosphere in radio ranging satellite. The Use of Artificial Satellites for Geodesy, 15, 247-251

Yang, F., Guo, J., Meng, X., Shi, J., Zhang, D., \& Zhao, Y. (2020a). An improved weighted mean temperature (Tm) model based on GPT2w with T m lapse rate. GPS Solutions, 24(2), 1-13. https://doi.org/10.1007/ s10291-020-0953-9

Yang, F., Guo, J., Zhang, C., Li, Y., \& Li, J. (2021). A regional zenith tropospheric delay (ZTD) model based on GPT3 and ANN. Remote Sensing, 13(5), 838. https://doi.org/10.3390/rs13050838.

Yang, H., Hu, W., Yu, L., Nie, X., \& Li, H. (2020c). GHop: a new regional tropospheric zenith delay model. Geomatics and Information Science of Wuhan University, 45(2), 226-232. https://doi.org/10.13203/j.whugis20180167.

Yang, L., Gao, J., Zhu, D., Zheng, N., \& Li, Z. (2020b). Improved zenith tropospheric delay modeling using the piecewise model of atmospheric refractivity. Remote. Sensing, 12, 3876. https://doi.org/10.3390/rs12233876.

Yang, T., Wan, W., Sun, Z., Liu, B., Li, S., \& Chen, X. (2020d). Comprehensive evaluation of using TechDemoSat-1 and CYGNSS data to estimate soil moisture over mainland China. Remote Sensing, 12, 1699. https://doi.org/ 10.3390/rs12101699.

Yao, Y., He, C., \& Zhang, B. (2013). A new global zenith tropospheric delay model GZTD. China Journal of Geophysics, 56, 2218-2227. https://doi.org/ 10.6038/cjg2013a0709.

Yao, Y., Hu, Y., Yu, C., Zhang, B., \& Guo, J. (2016). An improved global zenith tropospheric delay model GZTD2 considering diurnal variations. Nonlinear Process. Geophysics, 23, 127-136. https://doi.org/10.5194/ npg-23-127-2016.

Yao, Y., Sun, Z., \& Xu, C. (2018). Establishment and evaluation of a new meteorological observation-based grid model for estimating zenith wet delay in ground-based Global Navigation Satellite System (GNSS). Remote Sensing, 10(11), 1718. https://doi.org/10.3390/rs10111718.

Zhang, H., Yuan, Y., Li, W., Zhang, B., \& Ou, J. (2017). A grid-based tropospheric product for China using a GNSS network. Journal of Geodesy, 92, 765-777. https://doi.org/10.1007/s00190-017-1093-z

Zheng, F., Lou, Y., Gu, S., Gong, X., \& Shi, C. (2018). Modeling tropospheric wet delays with national GNSS reference network in China for BeiDou precise point positioning. Journal of Geodesy, 92, 545-560. https://doi.org/10. 1007/s00190-017-1080-4.

Zumberge, J., Heflin, M., Jefferson, D., Watkins, M., \& Webb, F. (1997). Precise point positioning for the efficient and robust analysis of GPS data from large networks. Journal of Geophysical Research: Atmospheres, 102, 5005-5017. https://doi.org/10.1029/96jb03860.

\section{Publisher's Note}

Springer Nature remains neutral with regard to jurisdictional claims in published maps and institutional affiliations.

\section{Submit your manuscript to a SpringerOpen ${ }^{\odot}$ journal and benefit from:}

- Convenient online submission

- Rigorous peer review

- Open access: articles freely available online

- High visibility within the field

- Retaining the copyright to your article

Submit your next manuscript at $\boldsymbol{\nabla}$ springeropen.com 